\title{
Editorial:
}

\section{Chronic kidney diseases in India - a ray of hope}

India, like the developed and many other developing countries, has witnessed a sharp rise in the incidence of chronic diseases and resultant mortality during the past few decades. This increasing burden of chronic diseases along with existing burden of communicable diseases is putting a strain on an already stretched health services of the country. This is leading to not only an increase in economic cost, but also an increase in the loss of quality adjusted life years (QALY). Till now in India, this has been reasonably documented with respect to cancer, diabetes, stroke and cardiovascular disease which led to initiation of national programs on prevention and control of these diseases [National Program on Prevention and Control of Diabetes, Cancer, Cardiovascular Disease and Stroke (NPCDCS) ]. ${ }^{1}$ Thus diabetes, cardiovascular diseases and stroke are well recognized by the public, physicians and the government. However, chronic kidney disease (CKD) was till recently unrecognized as a major chronic disease responsible for economic load to the government. In 1998, World Health Organization mentioned the ten common causes of death in India and CKD never figured in that document. Similarly, if one looks into the undergraduate medical curriculum considered by Medical Council of India, there is no mention of CKD as one of the topics to be covered. But if we look into the absolute number of deaths due to $\mathrm{CKD}$, it can be realized that CKD will find a place in the first ten causes of death in India.

CKD is not a single disease but a group of diseases characterized by evidence of either low glomerular filtration rate (GFR) $\left(<60 \mathrm{~mL} / \mathrm{min} / 1.74 / \mathrm{m}^{2}\right.$ body surface area) or evidence of kidney disease with relatively preserved GFR, present for more than three months (to be chronic) in absence of reversible factors and mostly progressive in nature. There is controversy in the method of determination of GFR. In most centres it is done by calculation from serum creatinine value (eGFR) rather than by true measurement (mGFR). eGFR, though practical, is not reliable in all clinical situations. Evidence of kidney damage is mostly arrived at by demonstration of significant proteinuria but may also be supported by other urinary sediment abnormalities or abnormalities in renal imaging. Of the approximate 1 million people in the world with severe CKD (also called end stage renal disease) who are being treated with some form of renal replacement therapy (RRT), 90\% live in developed countries. In India, of the approximate 150,000 new ESRD patients every year, only 5\%-10\% get some form of RRT. ${ }^{2}$ Outcome of the rest can easily be interpreted. Thus, it is clear that India cannot afford to manage all patients of ESRD. Hence, prevention of CKD evolves as a crucial issue not only for the medical fraternity but also for the government. Diabetes and hypertension constitute approximately $60 \%$ of all the causes of CKD. Both the diseases are also easy to diagnose, follow and treat, provided there is commitment to do so at every step i.e., physician, policy makers, government and the patient. It is necessary to dissipate this information at every forum we have at our hand.

It is obvious that in a country like India, screening the whole population for CKD is neither required nor possible. Thus, we need to concentrate on screening high-risk group for CKD. In addition to patients of diabetes and hypertension, who need regular screening for kidney involvement, first degree relatives of patients with CKD is one such high risk group that needs attention. In fact, this group is likely to be more receptive to the idea of screening as they have seen the suffering of a 
family member with CKD. Soumita et $\mathrm{al}^{3}$ have shown that such opportunistic screening of first degree relative does show a higher prevalence of CKD and its risk factors in family members. Such type of screening is possible at multiple levels of medical care in the society, starting from primary health centre to tertiary health centre.

In the last few years there has been a significant improvement in the awareness resulting in increasing efforts both at national and international levels to do advocacy and action in relation to CKD. At national level, Government of India has included CKD as one of the components of noncommunicable disease (NCD) program activity for the current five year plan. In August 2011, a national consultation held at Delhi for NCDs has ultimately produced a document titled "Call for action" and included CKD as one of the components of NCD requiring planning and action towards control and management. ${ }^{4}$ Department of Biotechnology (DBT), Government of India for the first time advertised call for research proposals in relation to CKD and supporting funding for the research projects related to CKD. However, there are still some steps to be taken like including CKD in Government of India initiated NCD clinics at community health centre and district level hospitals for control and prevention of non-communicable diseases. At international level, Asian Forum for CKD Initiative is working in various aspects of CKD prevention and management. ${ }^{5}$ International Society of Nephrology has recently made a CKD group, including Indian nephrologists, for the advocacy of CKD in different countries. With all these activities going on simultaneously, it looks that there is going to be a ray of hope for a concentrated effort towards effective management and prevention of CKD in India.

Sanjay K Agarwal

Professor and Head Department of Nephrology All India Institute of of Medical Sciences New Delhi, India e-mail: skagarwalnephro@gmail.com

Received: 7 May, 2012.

Agarwal SK. Chronic kidney diseases in India - a ray of hope. J Clin Sci Res 2012;3:112-3.

\section{REFERENCES}

1. National Programme for Prevention and Control of Cancer, Diabetes, Cardiovascular Diseases \& Stroke (NPCDCS). Available at URL: http://health.bih.nic.in/Docs/Guidelines-NPCDCS.pdf. Accessed on June 14, 2012.

2. Agarwal SK, Srivastava RK. CKD in India: challenges and solutions. Nephron Clin Pract 2009;111:c197-203.

3. Bagchi S, Agarwal SK, Gupta S. Targeted screening of adult first-degree relatives for chronic kidney disease and its risk factors. Nephron Clin Prac 2010;116:c128-36.

4. New Delhi Call for Action on combating Noncommunicable Diseases in Inida. Outcome of National NCD Summit jointly organized by Directorate General of Health Services, Ministry of Health \& Family Welfare, Govt. of India \& WHO country office for India, Vigyan Bhavan, New Delhi, 23-24 August 2011. Available at URL http://www.world-heart-federation.org/fileadmin/user_upload/documents/Advocacy/Resources/ Articles_Series_Reports/NewDelhi_NCDs_CallforAction.pdf. Accessed on June 14, 2012.

5. Tsukamoto Y. The mission of the Asian Forum of CKD Initiative (AFCKDI). Available at URL: http://www.asianpacific-ckd.com. Accessed on June 12, 2012. 